\section{BRAZIULIAN JOURNAL \\ OF MEDICAL AND BIOLOGICAL RESEARCH}

www.bjournal.com.br
ISSN 1414-431X

Volume 45 (12) 1102-1340 December 2012

\section{BIOMIDICAL SCIENCES}

AND

CLINICAL INVESTIGATION

Braz J Med Biol Res, December 2012, Volume 45(12) 1215-1220

doi: 10.1590/S0100-879X2012007500138

Resistance exercise improves hippocampus-dependent memory

R.C. Cassilhas, K.S. Lee, D.P. Venâncio, M.G.M. Oliveira, S. Tufik and M.T. de Mello

The Brazilian Journal of Medical and Biological Research is partially financed by

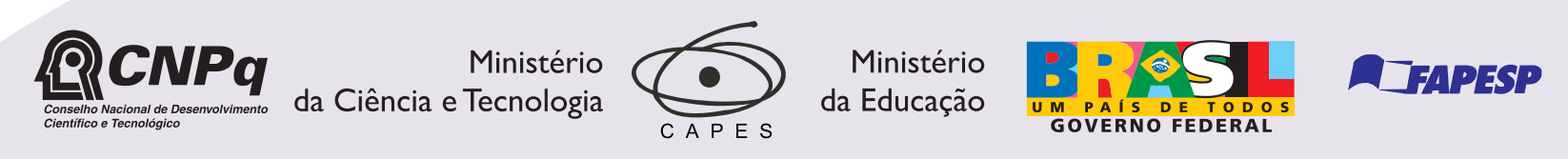

Institutional Sponsors

Sciezo
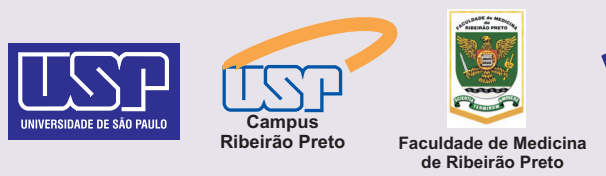

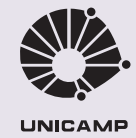

UNICAMP $\oplus$ SHIMADZU

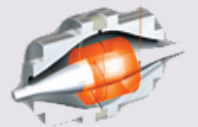

1DID Associaçăo Explore High - Performance MS Orbitrap Technology In Proteomics \& Metabolomics analitica Thermo 


\title{
Resistance exercise improves hippocampus-dependent memory
}

\author{
R.C. Cassilhas ${ }^{1,2}$, K.S. Lee ${ }^{3}$, D.P. Venâncio², M.G.M. Oliveira², \\ S. Tufik ${ }^{2}$ and M.T. de Mello ${ }^{1,2}$
}

${ }^{1}$ Centro de Estudos em Psicobiologia e Exercício, Universidade Federal de São Paulo, São Paulo, SP, Brasil

${ }^{2}$ Departamento de Psicobiologia, Universidade Federal de São Paulo, São Paulo, SP, Brasil ${ }^{3}$ Departamento de Bioquímica, Universidade Federal de São Paulo, São Paulo, SP, Brasil

\begin{abstract}
It has been demonstrated that resistance exercise improves cognitive functions in humans. Thus, an animal model that mimics this phenomenon can be an important tool for studying the underlying neurophysiological mechanisms. Here, we tested if an animal model for resistance exercise was able to improve the performance in a hippocampus-dependent memory task. In addition, we also evaluated the level of insulin-like growth factor 1/insulin growth factor receptor (IGF-1/IGF-1R), which plays pleiotropic roles in the nervous system. Adult male Wistar rats were divided into three groups $(\mathrm{N}=10$ for each group): control, SHAM, and resistance exercise (RES). The RES group was submitted to 8 weeks of progressive resistance exercise in a vertical ladder apparatus, while the SHAM group was left in the same apparatus without exercising. Analysis of a cross-sectional area of the flexor digitorum longus muscle indicated that this training period was sufficient to cause muscle fiber hypertrophy. In a step-through passive avoidance task (PA), the RES group presented a longer latency than the other groups on the test day. We also observed an increase of 43 and $94 \%$ for systemic and hippocampal IGF-1 concentration, respectively, in the RES group compared to the others. A positive correlation was established between PA performance and systemic IGF-1 ( $r=0.46$, $\mathrm{P}<0.05)$. Taken together, our data indicate that resistance exercise improves the hippocampus-dependent memory task with a concomitant increase of IGF-1 level in the rat model. This model can be further explored to better understand the effects of resistance exercise on brain functions.
\end{abstract}

Key words: Physical exercise; Passive avoidance; Insulin-like growth factor 1; Resistance training; Hippocampus; Memory

\section{Introduction}

The recent literature has reported that resistance exercise may be a promising intervention for some chronic diseases. For instance, bone mineral density, power, muscle mass, muscle strength, and functional abilities can be enhanced by this type of exercise (1). In addition, a number of studies performed in humans have suggested that resistance training improves several aspects of cognition (2-5).

Our group showed that resistance training for 24 weeks induced explicit long-term memory, working memory and executive function in elderly individuals with a significant increase in systemic insulin-like growth factor 1 (IGF-1) levels (5). A similar increase of systemic IGF-1 in elderly subjects submitted to resistance training has also been reported in another study (6). Moreover, peripheral IGF-1 level appears to positively correlate with cognitive perfor- mance, especially in the elderly population $(7,8)$.

These findings suggest that circulating IGF-1 contributes to the neuronal plasticity induced by resistance training. Indeed, studies have shown that peripheral IGF-1 can cross the brain blood barrier and play roles as a neurotrophic factor, particularly in the hippocampus, activating various central mechanisms related to cognitive processes (9). In addition to the systemic source, locally expressed IGF-1 and its receptor (IGF-1R) in the hippocampus can also play a part in neuroplastic changes induced by voluntary physical exercise as shown by Ding et al. (10).

Whereas the positive impact of resistance exercise on cognitive functions is evident in humans, its driving neurophysiological mechanisms remain unclear, although IGF-1 appears to be one of the mediators. Thorough investigation of these beneficial effects may not only bring new insight

Correspondence: M.T. Mello, Rua Francisco de Castro, 93, 04020-050 São Paulo, SP, Brasil. Fax: +55-11-5572-0177.

E-mail: tmello@demello.net.br

Received March 1, 2012. Accepted August 14, 2012. Available online August 31, 2012. Published December 17, 2012. 
into the communication between central nervous system and body parts, but may also provide valuable information for the development of novel therapeutic strategies for neurological diseases (11).

Improved muscle strength and hypertrophy observed in humans after resistance exercise can be reproduced in rodent models using several distinct apparatuses for resistance exercise (12). However, the effect of resistance exercise on brain functions has not been evaluated in these models. Thus, the objective of the present study was to validate an animal model for resistance exercise (in rats), capable of improving the performance in hippocampusdependent memory tasks and also to correlate memory task performance with IGF-1/IGF-1R levels.

\section{Material and Methods}

All animal procedures followed the National Institutes of Health Guide for the Care and Use of Laboratory Animals (Publication No. 85-23, revised 1985), approved by the Ethics Committee of UNIFESP (License No. 0778/07) and were performed under adherence to the American College of Sports Medicine animal care standards.

\section{Animal care}

Wistar rats, 90 days old, were provided by the "Centro de Desenvolvimento de Modelos Experimentais para Medicina e Biologia" (CEDEME), Universidade Federal de São Paulo. The animals were housed in groups of 5 in standard polypropylene cages. Room temperature was maintained at $22^{\circ} \pm 1^{\circ} \mathrm{C}$ and relative humidity at $55 \pm 3 \%$. Animals were kept on a 12:12-h light-dark schedule (lights on at 7:00 am) and had free access to food and water. All experimental procedures were performed between 1:00 and 5:00 pm according to established guidelines (13).

\section{Familiarization protocol: up ladder performance}

Before any manipulations or resistance training, all animals were submitted to a familiarization session named "up ladder performance" on an $80^{\circ}$ inclined vertical ladder apparatus (110 x $18 \mathrm{~cm}$ ) with 2-cm grid steps. A housing chamber $(25 \times 25$ x $20 \mathrm{~cm}$ ) was located at the top of the ladder $(12,14)$.

The familiarization protocol consisted of three trials per day for 3 days. During this protocol, the rats were kept in the housing chamber for $60 \mathrm{~s}$, and then placed on the proximal length $(35 \mathrm{~cm})$ of the ladder. In the second trial, the rats were placed on the middle length $(55 \mathrm{~cm})$ of the ladder. In the last trial, the rats climbed the whole ladder $(110 \mathrm{~cm})$, and the time spent climbing was recorded. According to this up ladder performance, the animals were paired and distributed into the following groups: control group (CTRL, $N=10$ ), sham group (SHAM, N = 10), and resistance exercise group (RES, $N=10$ ). This protocol assures that the animals are homogenously distributed into three groups, at least in relation to climbing performance.

\section{Control group}

CTRL animals were left in their home cages throughout the experiment and were handled for 5 min daily, 5 times a week (Monday to Friday).

\section{SHAM group}

SHAM animals were left at the top of the vertical ladder for 15 min while RES animals exercised on the same apparatus. This procedure was performed 5 times per week (Monday to Friday). This group was included to evaluate possible effect of animal exposure to a novel environment (vertical ladder apparatus).

\section{Resistance exercise protocol}

The resistance training protocol was modified from the previously described one $(12,14)$. RES animals were submitted to progressive resistance exercise for 8 weeks, 5 times a week (Monday to Friday) using a load that consisted of $50-\mathrm{mL}$ conical tubes attached to $13.5-\mathrm{cm}$ plastic-coated steel cable fixed by a coastlock snap swivel. The load was fixed on the tail of the rats using a Scotch 23 rubber tape (Scotch 3M, USA). For each climb, the animals had to make 8-12 repetitive dynamic movements to reach the housing chamber. The resistance exercise protocol is presented in Table S1.

\section{Passive avoidance task}

Twenty-four hours after the last training session, the animals were submitted to a step-through passive avoidance (PA) task. The PA apparatus consisted of two acrylic boxes, each measuring $21 \times 26 \times 27.5 \mathrm{~cm}$, connected by a sliding door. A box with white acrylic walls was designated as the safe compartment, whereas the other black acrylic box was the aversive compartment. The floor of the apparatus consisted of parallel metal rods $(0.4 \mathrm{~cm}$ in diameter $)$, separated by a distance of $1.2 \mathrm{~cm}$ from each other, and connected to an electric shock generator. During the task training, each animal was placed in the safe compartment with the sliding door closed. Ten seconds later, the door was opened. As soon as the animal crossed to the aversive compartment with its four paws, the door was closed and the animal received one footshock $(0.6 \mathrm{~mA} / 1 \mathrm{~s})$. The latency to cross into the black chamber was measured in seconds. In the test phase, $24 \mathrm{~h}$ later, the animals were subjected to the same test and the latency to move to the aversive chamber was measured $(15,16)$. The maximal latency was set at $540 \mathrm{~s}$.

\section{Tissue collection and processing}

Twenty-four hours after the memory task, the rats were euthanized by decapitation. The trunk blood was collected into dry tubes and centrifuged (3500 g, $10 \mathrm{~min}$ ) for serum collection. The hippocampus and the striated skeletal muscle flexor digitorum longus muscle (FDL, an ankle extensor muscle that participates in the climbing move- 
ment) of the right leg were dissected. The hippocampus was immediately frozen in liquid nitrogen, and each FDL muscle was entirely removed and coated with embedding medium (Tissue-Tek OCT, Miles Inc., USA) and immersed in liquid nitrogen-cooled isopentane. All tissues and serum were stored at $-80^{\circ} \mathrm{C}$ until use.

\section{Measurement of fiber cross-sectional area of the FDL muscle}

Transverse sections ( $8-\mu \mathrm{M}$ thick) were cut from the midbelly of the flexor muscle in a cryostat at $-20^{\circ} \mathrm{C}$, melted onto poly-L-lysine coated microscopic slides (Superfrost, Fisher Scientific, USA) and stained with Meyer's hematoxylineosin (H\&E). Digital images of H\&E-stained sections were obtained with an Olympus bright field microscope BX50, fitted with a DP71 camera (USA) with a 40X objective. Blind analysis of the cross-sectional area of 100 fibers per muscle sample was performed using the Axio Vision 4.6 software (Carl Zeiss Microlmaging $\mathrm{GmbH}$, Germany).

\section{IGF-1 measurement}

The hippocampus was homogenized as described elsewhere (17) and the total protein content was determined by the method of Lowry et al. (18). The concentration of systemic and hippocampal IGF-1 was measured (in serum) using an immunoradiometric assay kit for IGF-1 (IRMAIGF-1 A15729, Immunotech, France) according to manufacturer specifications. Human recombinant IGF-1 was provided for the standard curve, which was not appropriate for rat samples. Thus, the IGF-1 levels in the SHAM and RES groups are reported as percentage of the control mean.

\section{Statistical analysis}

The Statistica software (Statsoft, USA) was used for all statistical analyses. The Shapiro-Wilk W-test was used to determine normality. For the parametric variables (latency on the day of the PA test; muscle fiber cross-sectional area), one-way ANOVA was used, followed by the Tukey test. The Kruskal-Wallis test was used for the nonparametric variables (systemic and hippocampal IGF-1 and hippocampal IGF1R). The Spearman correlation coefficient between PA performance and systemic IGF1 was calculated. The level of significance was set at $5 \%$, and data are reported as means $\pm \mathrm{SD}$.

\section{Results}

\section{Effect of progressive resistance exercise on FDL muscle}

To ensure the effectiveness of the exercise, we analyzed the cross-sectional area of the FDL muscle. As shown in Figure 1, the muscle fiber area of the RES group was increased compared to SHAM and CTRL [CTRL $=2753.80 \pm 359.54$ $\mu \mathrm{m}^{2} ; \mathrm{SHAM}=2620.57 \pm 205.93 \mu \mathrm{m}^{2} ; \mathrm{RES}=3711.84 \pm$ $279.45 \mu \mathrm{m}^{2} ; F_{(2,27)}=42.56, \mathrm{P} \leq 0.001$ ], indicating the efficacy of the protocol applied for 8 weeks to cause muscle hypertrophy.

\section{Effect of progressive resistance exercise on the memory task}

Figure 2 shows the results of the inhibitory avoidance

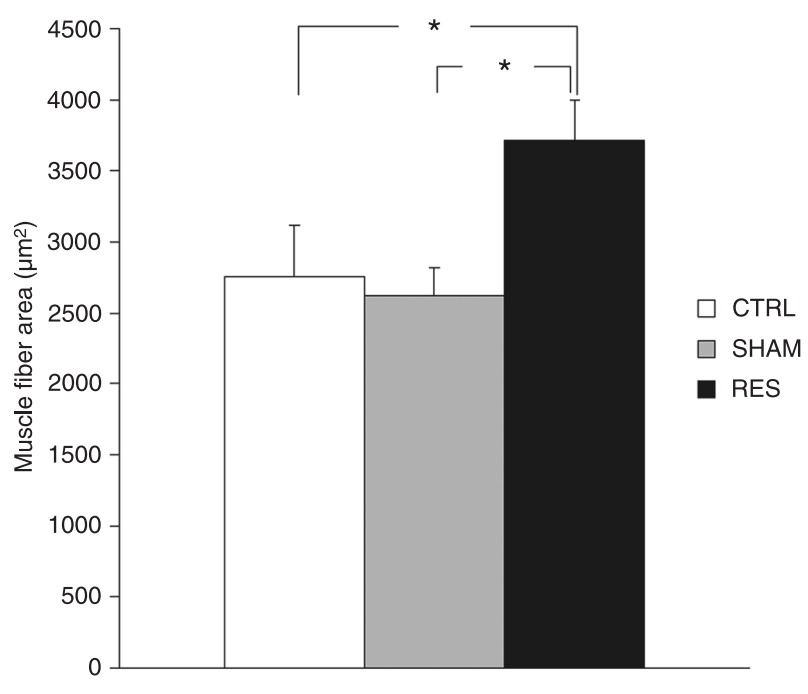

Figure 1. Effect of resistance exercise on the cross-sectional area of the rat flexor digitorum longus (FDL) muscle. The FDL muscle fiber cross-sectional area of control (CTRL, $N=10$ ), sham (SHAM, $N=10$ ), and resistance exercise groups (RES, $N$ $=10$ ) was measured after 8 weeks of exercise. Data are reported as means $\pm \mathrm{SD}$. ${ }^{*} \mathrm{P} \leq 0.001$ (one-way ANOVA followed by the Tukey test).

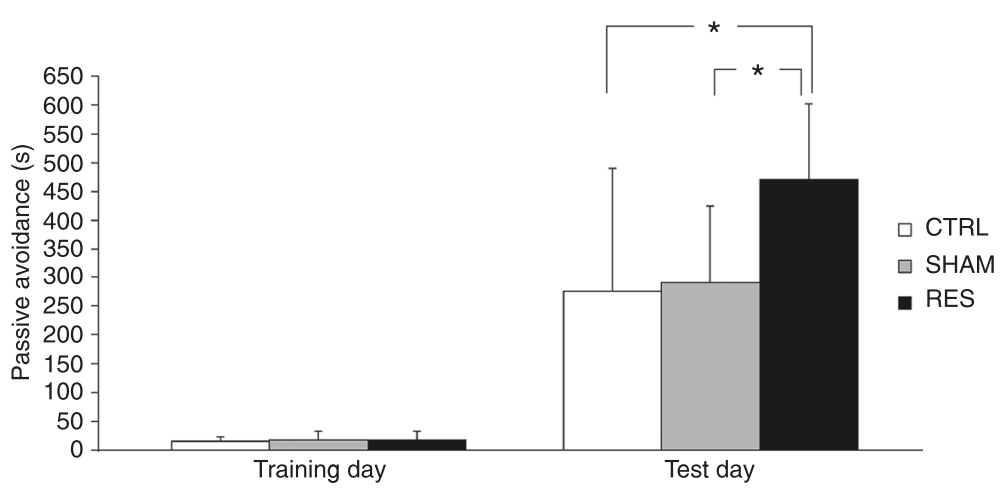

Figure 2. Effect of resistance exercise on the passive avoidance test. Latency to enter the dark compartment on the training day and test day of control (CTRL, N $=10$ ), sham (SHAM, $N=10$ ), and resistance exercise groups (RES, $N=10$ ) was measured after 8 weeks of exercise. Data are reported as means \pm SD in relation to the CTRL group. ${ }^{*} \mathrm{P} \leq 0.001$ (Kruskal-Wallis test). 
test, measured by latency to enter the dark compartment on the test day, indicating an improvement of memory in the RES group compared to the other groups [CTRL $=275.50$ $\pm 214.61 \mathrm{~s} ;$ SHAM $=291.60 \pm 134.02 \mathrm{~s} ;$ RES $=470.30 \pm$ $\left.132.48 \mathrm{~s} ; F_{(2,27)}=4.64, \mathrm{P} \leq 0.05\right]$.

\section{Effect of the progressive resistance exercise on IGF-1 levels}

As shown in Figure $3 A$ and $B$, the relative concentration of systemic and hippocampal IGF-1 was increased in the RES group compared to the CTRL and SHAM groups [Figure $3 A$ : CTRL $=100 \pm 5.81 \%$; SHAM $=95.92 \pm 7.12 \%$; RES $=143.40 \pm 14.89 \% ; \mathrm{H}=20.39, \mathrm{P} \leq 0.001]$. [Figure 3B: CTRL = $100 \pm 14.77 \%$; SHAM = $104 \pm 20.05 \% ;$ RES $=194.64 \pm 27.76 \% ; \mathrm{H}=$ $19.59, \mathrm{P} \leq 0.001]$. Systemic IGF-1 showed a positive correlation with the latency to cross into the dark compartment on the day of the PA test (Figure 4; $r=0.46 ; P \leq 0.05$ ). Moreover, the concentration of systemic IGF-1 was correlated with hippocampal IGF-1 (Figure 5; r $=0.83 ; \mathrm{P} \leq 0.001)$, suggesting a relationship between systemic and hippocampal IGF-1.

\section{Discussion}

Resistance training, a type of physical exercise to increase muscle mass, strength by doing repetitive exercises with weights, weight machines, or resistance bands, is one of the most recommended exercises and is applied as a primary intervention for sarcopenia, improvement of physical function, and prevention of functional limitations and muscle weakness in elderly persons (19). In addition, there is growing evidence that resistance exercise may be a positive factor for cognitive functions $(2,5,20)$. Thus, an animal model that mimics the effects of resistance exercise may be an important tool to understand the underlying mechanisms.

The increase of muscle size is referred to as hypertrophy, and this adaptation is one of the most important features associated with resistance exercise training. In the present study, the RES group, which performed progressive resistance training, exhibited FDL muscle fiber hypertrophy. This result confirms the efficacy of the exercise protocol and corroborates other studies that found similar results $(12,14)$.

After the intervention period, the RES group took more time to enter the dark compartment on the test day in the inhibitory avoidance task compared to the CTRL and SHAM groups. Similar memory improvement has also been observed in animals submitted to forced treadmill running, swimming or voluntary wheel running protocols, where aerobic metabolism is emphasized $(10,21-28)$. To the best of our knowledge, ours is the first study that shows the positive impact of progressive resistance exercise on memory using an animal model. Moreover, the properties of this animal model not only reproduced our previous observation showing induction of serum IGF-1 after resistance training in humans (5), but also demonstrated that hippocampal and systemic IGF-1 increased concomitantly. Similar results were observed in clinical studies showing
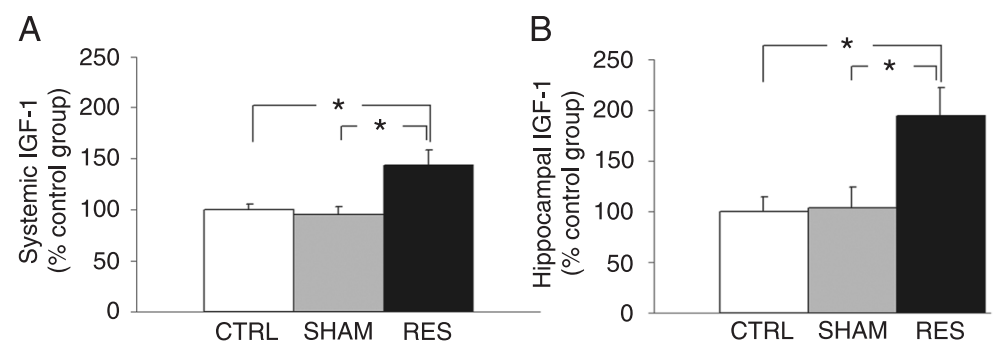

Figure 3. Systemic and hippocampal insulin-like growth factor-1 (IGF-1, ng/mL). The concentration of systemic $(A)$ and hippocampal $(B)$ IGF-1 of control (CTRL, $N=10$ ), sham (SHAM, $N=10$ ), and resistance exercise groups (RES, $N=10$ ) was measured after 8 weeks of exercise. Data are reported as means \pm SD in relation to the CTRL group. ${ }^{*} \mathrm{P} \leq 0.001$ (Kruskal-Wallis test)

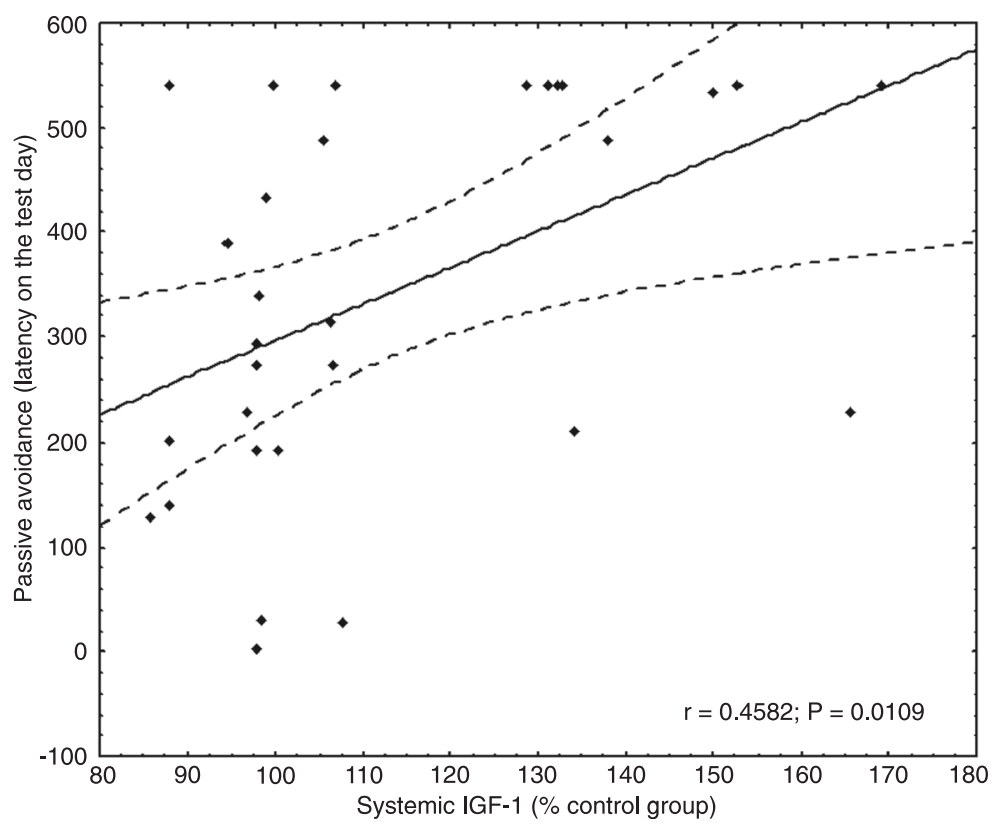

Figure 4. Correlation between the concentration of systemic insulin-like growth factor-1 (IGF-1, ng/mL) and the latency to enter the dark compartment of the passive avoidance apparatus on the test day. The Spearman rank order correlation test was performed using samples from all groups $(N=30)$. 
that young $(29,30)$ or elderly people $(31,32)$ submitted to a resistance protocol had increased systemic IGF-1.

It is well known that blood IGF-1 can cross the blood-brain barrier (33), activate the IGF-1/IGF-1R signaling pathway (34), influence the hippocampal structure and improve cognition and neuroplasticity (35). The positive correlations between performance in the PA task and systemic IGF-1 levels and between hippocampal and systemic IGF-1 levels reinforce the idea that circulating IGF-1 has an important role in mediating the effects of resistance exercise on the brain. In fact, the blockage of peripheral IGF-1 prevents running-induced adult neurogenesis, cell proliferation in the dentate gyrus and spine density in basal dendrites of CA1 pyramidal cells $(36,37)$. This crosstalk between peripheral and central compartments makes the IGF-1/IGF-1R signaling pathway an interesting therapeutic target for neurological disorders (35).

The memory improvement observed in humans after resistance training was reproduced in a rat model with a concomitant increase of systemic and hippocampal IGF-1. This rat model of resistance training opens a new avenue of research in neuroscience related to sports medicine and exercise.

\section{Supplementary material}

Table S1.

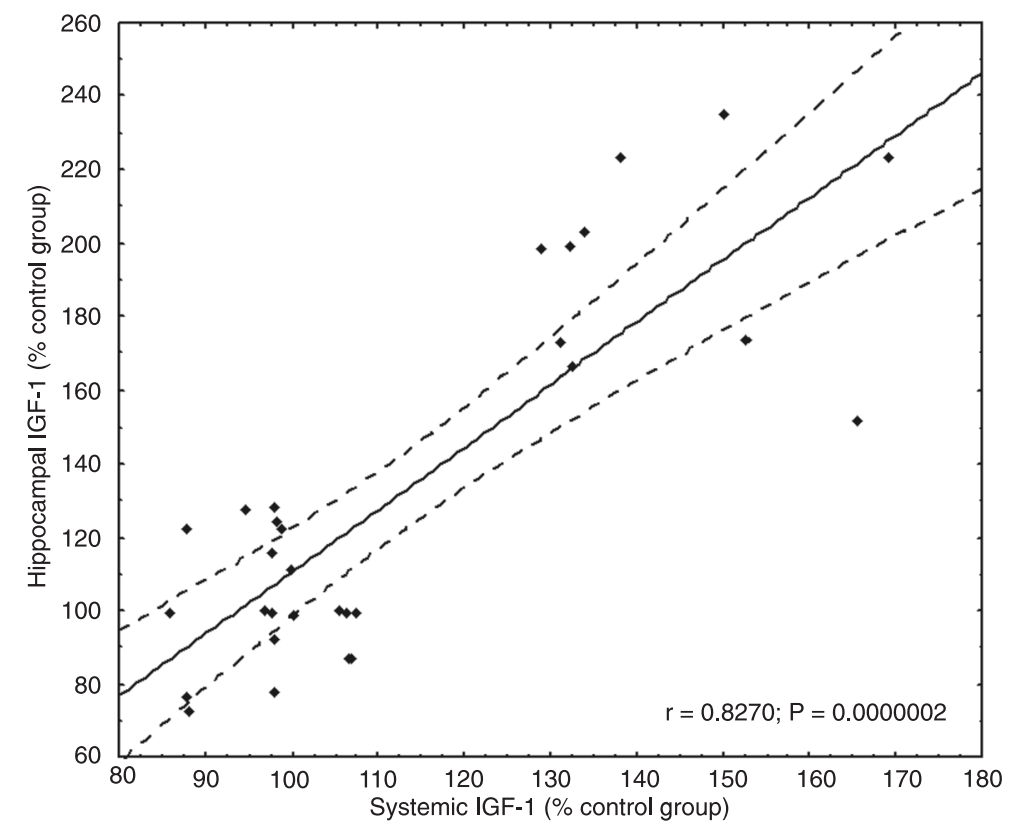

Figure 5. Correlation between systemic and hippocampal insulin-like growth factor-1 (IGF-1, ng/mL). The Spearman rank order correlation test was performed using samples from all groups $(\mathrm{N}=30)$.

\section{Acknowledgments}

Research supported by FAPESP (\#2008/03083-6), CNPq, Associação Fundo de Incentivo à Pesquisa (AFIP), Centro de Estudos em Psicobiologia e Exercício (CEPE), Centro de Estudo Multidisciplinar em Sonolência e Acidentes (CEMSA), and Centro de Pesquisa, Inovação e Difusão (CEPID-Sleep).

\section{References}

1. Haskell WL, Lee IM, Pate RR, Powell KE, Blair SN, Franklin $B A$, et al. Physical activity and public health: updated recommendation for adults from the American College of Sports Medicine and the American Heart Association. Med Sci Sports Exerc 2007; 39: 1423-1434.

2. Liu-Ambrose T, Nagamatsu LS, Graf P, Beattie BL, Ashe MC, Handy TC. Resistance training and executive functions: a 12-month randomized controlled trial. Arch Intern Med 2010; 170: 170-178.

3. Özkaya GY, Aydýn H, Toraman FN, Kýzýlay F, Cetinkaya $V$. Effect of strength and endurance training on cognition in older people. J Sports Sci \& Med 2005; 4: 300-313.

4. Perrig-Chiello P, Perrig WJ, Ehrsam R, Staehelin HB, Krings $F$. The effects of resistance training on well-being and memory in elderly volunteers. Age Ageing 1998; 27: 469-475.

5. Cassilhas RC, Viana VA, Grassmann V, Santos RT, Santos $\mathrm{RF}$, Tufik S, et al. The impact of resistance exercise on the cognitive function of the elderly. Med Sci Sports Exerc 2007; 39: 1401-1407.

6. Borst SE, De Hoyos DV, Garzarella L, Vincent K, Pollock

$\mathrm{BH}$, Lowenthal DT, et al. Effects of resistance training on insulin-like growth factor-I and IGF binding proteins. Med Sci Sports Exerc 2001; 33: 648-653.

7. Aleman A, Verhaar HJ, de Haan EH, De Vries WR, Samson $\mathrm{MM}$, Drent ML, et al. Insulin-like growth factor-I and cognitive function in healthy older men. J Clin Endocrinol Metab 1999; 84: 471-475.

8. Dik MG, Pluijm SM, Jonker C, Deeg DJ, Lomecky MZ, Lips P. Insulin-like growth factor I (IGF-I) and cognitive decline in older persons. Neurobiol Aging 2003; 24: 573-581.

9. Trejo JL, Piriz J, Llorens-Martin MV, Fernandez AM, Bolos $M$, LeRoith $D$, et al. Central actions of liver-derived insulinlike growth factor I underlying its pro-cognitive effects. Mol Psychiatry 2007; 12: 1118-1128.

10. Ding Q, Vaynman S, Akhavan M, Ying Z, Gomez-Pinilla F. Insulin-like growth factor I interfaces with brain-derived neurotrophic factor-mediated synaptic plasticity to modulate aspects of exercise-induced cognitive function. Neuroscience 2006; 140: 823-833.

11. Arpa J, Sanz-Gallego I, Medina-Baez J, Portela LV, Jardim 
LB, Torres-Aleman I, et al. Subcutaneous insulin-like growth factor-1 treatment in spinocerebellar ataxias: an open label clinical trial. Mov Disord 2011; 26: 358-359.

12. Prestes J, de Cassia MR, Shiguemoto GE, Leite RD, Pereira GB, Selistre-de-Araujo HS, et al. Effects of ovariectomy and resistance training on MMP-2 activity in skeletal muscle. Appl Physiol Nutr Metab 2009; 34: 700-706.

13. Andersen ML, Tufik S. Animal models as ethical tools in biomedical research. 1st edn. São Paulo: CLR Balieiro Editores; 2010.

14. Hornberger TA Jr, Farrar RP. Physiological hypertrophy of the FHL muscle following 8 weeks of progressive resistance exercise in the rat. Can J Appl Physiol 2004; 29: 16-31.

15. Moreira KM, Hipolide DC, Nobrega JN, Bueno OF, Tufik S, Oliveira MG. Deficits in avoidance responding after paradoxical sleep deprivation are not associated with altered $\left[{ }^{3} \mathrm{H}\right]$ pirenzepine binding to $\mathrm{M} 1$ muscarinic receptors in rat brain. Brain Res 2003; 977: 31-37.

16. Rosa EF, Takahashi S, Aboulafia J, Nouailhetas VL, Oliveira MG. Oxidative stress induced by intense and exhaustive exercise impairs murine cognitive function. $J$ Neurophysiol 2007; 98: 1820-1826.

17. Ozguner IF, Savas C, Ozguner M, Delibas N. Effects of nonstrangulated small bowel obstruction on intestinal histology, insulin-like growth factor-I level, antioxidants, and lipid peroxidation in rats. Saudi Med J 2006; 27: 405-407.

18. Lowry $\mathrm{OH}$, Rosebrough NJ, Farr AL, Randall RJ. Protein measurement with the Folin phenol reagent. $J$ Biol Chem 1951; 193: 265-275.

19. Thomas DR. Sarcopenia. Clin Geriatr Med 2010; 26: 331346.

20. Chang YK, Etnier JL. Exploring the dose-response relationship between resistance exercise intensity and cognitive function. J Sport Exerc Psychol 2009; 31: 640-656.

21. Alaei $H$, Moloudi $R$, Sarkaki AR. Effects of treadmill running on mid-term memory and swim speed in the rat with Morris water maze test. J Bodyw Mov Ther 2008; 12: 72-75.

22. AsI NA, Sheikhzade F, Torchi M, Roshangar L, Khamnei S. Long-term regular exercise promotes memory and learning in young but not in older rats. Pathophysiology 2008; 15: 9-12.

23. Collins A, Hill LE, Chandramohan Y, Whitcomb D, Droste SK, Reul JM. Exercise improves cognitive responses to psychological stress through enhancement of epigenetic mechanisms and gene expression in the dentate gyrus. PLoS One 2009; 4: e4330.

24. Gobeske KT, Das S, Bonaguidi MA, Weiss C, Radulovic J, Disterhoft JF, et al. BMP signaling mediates effects of exercise on hippocampal neurogenesis and cognition in mice. PLoS One 2009; 4: e7506.
25. Ogonovszky H, Berkes I, Kumagai S, Kaneko T, Tahara $\mathrm{S}$, Goto S, et al. The effects of moderate-, strenuous- and over-training on oxidative stress markers, DNA repair, and memory, in rat brain. Neurochem Int 2005; 46: 635-640.

26. Radak Z, Toldy A, Szabo Z, Siamilis S, Nyakas C, Silye $G$, et al. The effects of training and detraining on memory, neurotrophins and oxidative stress markers in rat brain. Neurochem Int 2006; 49: 387-392.

27. van Praag H, Shubert T, Zhao C, Gage FH. Exercise enhances learning and hippocampal neurogenesis in aged mice. J Neurosci 2005; 25: 8680-8685.

28. Vaynman S, Ying Z, Gomez-Pinilla F. Exercise induces BDNF and synapsin I to specific hippocampal subfields. $J$ Neurosci Res 2004; 76: 356-362.

29. West DW, Kujbida GW, Moore DR, Atherton P, Burd NA, Padzik JP, et al. Resistance exercise-induced increases in putative anabolic hormones do not enhance muscle protein synthesis or intracellular signalling in young men. J Physiol 2009; 587: 5239-5247.

30. Rojas VS, Knicker A, Hollmann W, Bloch W, Struder HK. Effect of resistance exercise on serum levels of growth factors in humans. Horm Metab Res 2010; 42: 982-986.

31. Vale RG, de Oliveira RD, Pernambuco CS, de Meneses YP, Novaes JS, de Andrade AF. Effects of muscle strength and aerobic training on basal serum levels of IGF-1 and cortisol in elderly women. Arch Gerontol Geriatr 2009; 49: 343347.

32. Cassilhas RC, Antunes HK, Tufik S, de Mello MT. Mood, anxiety, and serum IGF-1 in elderly men given 24 weeks of high resistance exercise. Percept Mot Skills 2010; 110: 265-276

33. Carro E, Nunez A, Busiguina S, Torres-Aleman I. Circulating insulin-like growth factor I mediates effects of exercise on the brain. J Neurosci 2000; 20: 2926-2933.

34. Trejo JL, Carro E, Lopez-Lopez C, Torres-Aleman I. Role of serum insulin-like growth factor I in mammalian brain aging. Growth Horm IGF Res 2004; 14 (Suppl A): S39-S43.

35. Cassilhas RC, Lee KS, Fernandes J, Oliveira MG, Tufik S, Meeusen R, et al. Spatial memory is improved by aerobic and resistance exercise through divergent molecular mechanisms. Neuroscience 2012; 202: 309-317.

36. Trejo JL, Carro E, Torres-Aleman I. Circulating insulin-like growth factor I mediates exercise-induced increases in the number of new neurons in the adult hippocampus. J Neurosci 2001; 21: 1628-1634.

37. Glasper ER, Llorens-Martin MV, Leuner B, Gould E, Trejo JL. Blockade of insulin-like growth factor-I has complex effects on structural plasticity in the hippocampus. Hippocampus 2010; 20: 706-712. 